\title{
Cognitive Function, Aging, and the Community Dwelling Elderly Living Alone: Implications for Nursing Practice
}

\author{
Tanya L. Sleeper, PhD., MSN, MSB, RN \\ Professor, Department of Nursing Sciences, University of Maine, Fort Kent, United States.
}

\section{Article Details \\ Article Type: Research Article \\ Received date: $08^{\text {th }}$ July, 2021 \\ Accepted date: $26^{\text {th }}$ August, 2021 \\ Published date: $27^{\text {th }}$ August, 2021}

"Corresponding Author: Tanya L. Sleeper, PhD., MSN, MSB, RN, Professor, Department of Nursing Sciences, University of Maine at Fort Kent, United States. E-mail: tanya.sleeper@maine.edu

Citation: Sleeper, T. L., (2021). Cognitive Function, Aging, and the Community Dwelling Elderly Living Alone: Implications for Nursing Practice. J Comp Nurs Res Care 6(2):176. doi: https://doi.org/10.33790/jcnrc1100176.

Copyright: (C2021, This is an open-access article distributed under the terms of the Creative Commons Attribution License 4.0, which permits unrestricted use, distribution, and reproduction in any medium, provided the original author and source are credited.

\begin{abstract}
The growth in the aging population in the coming years represents a significant public health policy issue as more and more older adults wish to age in place. Additionally, as more older adults live longer, more older adults are living at home alone. Cognitive function and cognitive decline in later life represent additional challenges to the vulnerable elderly. As the health care system grapples to meet the needs of a growing aging population, the nursing profession can play an important role in health promotion and prevention efforts in cognition and aging. Given the need to advance more routine screening and assessment of cognition in later life, nurses are well poised to advance science and research and support recommendations for assessing cognitive function and cognitive decline in order to support safe and autonomous living in advanced age.
\end{abstract}

Key Words: Aging, Cognition, Cognitive Function, Nursing

\section{Introduction}

Assessment of cognitive capacity to determine incapacitation or dependence represents a complex process that is not easily measured or defined [1]. A myriad of contributing factors (including modifiable and non-modifiable factors), influence cognitive capacity and the ability to manage care [2]. Given the complexity of cognitive functioning, this article serves to raise awareness concerning the need to establish more robust guidelines and standards for providing more accurate assessments in determining and predicting older adults' capacity, particularly for those living alone in the community setting. Further, this article intends to leverage the findings from research to advocate for the use of screening tools and best practice approaches to enhance the assessment of cognitive capacity for older adults to provide better measures of cognitive function for the health care system to use when determining capacity to self-direct. Lastly, this article provides implications for nursing practice and their role in assessing cognitive capacity in older adults living alone in the community setting.

\section{Background}

The change in the demographic landscape signals an important shift concerning the characteristics of the older adult population and its implications for cognitive function in advanced age. The older adult population already represents a significant segment of the overall US population as growth in the aging population nationally is expected to increase drastically over the next several years. Adults 65 and older are expected to double in the next thirty years and comprise approximately $25 \%$ of the US population [3]. Additionally, older adults with advanced age are also living longer as advances in medicine and technology impact mortality and morbidity rates for persons living with acute and chronic diseases. Further, life expectancy has increased over time, with people living on average 78.7 years in 2018 [4]. The number of people aged 85 and older is expected to triple over the next thirty years and account for almost 20 million of the overall population [5].

Older adults living in rural America live longer; living alone poses additional challenges to the most vulnerable elderly. This phenomenon is particularly prevalent in the United States, where over a quarter of the older adult population, ages 60 and older, live alone [6]. The vulnerable community-dwelling older adult living alone poses additional risk related to the lack of support and social isolation for physical and cognitive decline impacting decisionmaking capacity [7]. An elder orphan is defined as "aged communitydwelling individuals who are socially and/or physically isolated, without an available known family member or designated surrogate or caregiver [8]. A recent study by the National Academies of Science, Engineering, and Medicine (NASEM) found that social isolation and loneliness, as a consequence of living alone, significantly increased the risk of chronic diseases, including dementia [9].

\section{Cognition and Aging}

Cognitive decline and cognitive capacity in advancing age are important considerations for older adults living alone. Adults ages 45 years or older living alone in the US, reporting a subjective cognitive decline (SCD), ranged from $19.7-52.2 \%$ nationally [10]. The presence of cognitive decline/cognitive impairment in older adults living alone resulted in additional concerns regarding their ability to live independently related to awareness of cognitive impairment, self-care management challenges, and a lack of available resources and supports [11]. Given these findings, as the older adult population grows and life expectancy increases, cognitive decline in advanced age in community-dwelling adults poses significant risks for the health and safety of those individuals living alone.

The assessment of cognitive function represents a complex multidimensional process influenced by many factors [12]. Cognition is characterized by several interdependent "domains of cognitive 
performance" that determine the overall capacity to perform various functions and skills necessary to manage care [13]. These domains encompass several functions, including sensation, perception, motor skills and construction, attention and concentration, memory, executive functioning, processing speeds, and language/verbal skills [13].

In particular, older adults are more prone to be exposed to factors influencing cognitive function attributable to age-related considerations and the presence of chronic disease. Given these increased risks, it is important to understand age-related changes in cognition and factors that positively and negatively affect cognitive function [14]. The National Institute on Aging stresses the importance of assessing cognitive function in older adults given the myriad of modifiable and non-modifiable factors that result in cognitive impairment, including the effects of medications, metabolic and endocrine disorders, acute and chronic disease, and the presence of depression and dementia [14]. In light of the vast array of factors that can influence cognitive function in advanced age, assessment of cognitive capacity should include tools and approaches to identify such factors.

Despite the growth in the aging population and the increased concerns surrounding cognitive decline, there continues to be a lack of consensus surrounding the benefits of cognitive screening for community-dwelling older adults [15]. While several assessment tools exist, these tools are not routinely used in clinical practice, particularly in patients presenting with conditions affecting cognition [16]. Further, health care professionals cite several barriers in practice that impact their ability to routinely conduct effective cognitive evaluations [17].

\section{Assessing Cognitive Capacity and Cognitive Decline}

The community-dwelling older adult living alone presents unique challenges and concerns regarding the assessment of cognitive capacity and their ability to live safely at home. Given the complexity of cognitive capacity, the need to assess and evaluate multiple domains of cognitive and executive functioning is necessary to self-direct and manage care in the solo community-dwelling elder [24].The nursing profession can play a critical role in assessing cognitive capacity in the community dwelling solo elder. Drawing from the profession's early roots, "public health nurses have been critical to the functioning of our health care system by improving the health of vulnerable populations and entire communities" [25]. Efforts to strengthen the public health infrastructure and help address the unique needs and challenges of a growing aging population are paramount.

Additionally, given the wide away of institutional, outpatient and community-based setting that nurses are employed, the profession is well poised to play a lead role in assessing cognitive capacity to advance health promotion/prevention efforts in older adults [26]. Specifically, drawing from current research, nurses educated in the field of aging can utilize standardized assessment tools and other recommendations to provide ongoing assessment of cognitive capacity in older adults in support of key recommendations from the NIH and others within the field [14, 21, 22]. As an integral member of the interdisciplinary health care team, a patient-centered approach to nursing care provides meaningful opportunities to enhance quality of life and associated outcomes in the older adult population [25].

\section{Conclusion}

The shift in the demographic landscape in response to the growth in the aging population and the number of older adults living at home alone constitutes a pressing policy issue. The need to address cognitive decline in advanced age already exceeds the current systems capacities to adequately assess and evaluate cognitive capacity. Further, gaps in practice fail to adequately assess and predict cognitive function to direct and manage care. Given the unique needs of the older adult living alone and the complexity of cognitive function, health care systems must be better equipped to ensure the accuracy, validity, and reliability of cognitive assessments and the ability to live as safely and autonomously at home. The nursing profession is well poised to fill that gap and leverage findings from research and other recommendations to assess cognitive capacity and enhance health promotion and prevention efforts for older adults living at home alone.

Conflict of interests: The author declares no conflict of interest.

\section{References}

1. Holly, Ramsey-Klawsnik. (2014). The Complexities of Cognitive Capacity. http://www.napsa-now.org/wp- content/ uploads/2015/06/TA-Brief-Mental-Capacity-FINAL.pdf

2. Darren M. Lipnicki, et al., (2020). Determinants of cognitive performance and decline in 20 diverse ethno-regional groups: A COSMIC collaboration cohort study." 16, Plos Med. (Jul. 23,2019)DOI: 10.1371/journal.pmed.1002853Population Reference Bureau, Fact Sheet - Aging in the US. https://www. prb.org/aging-unitedstates-fact-sheet/

3. Jiaquan, Xu., Sherry L. Murphy., Kenneth D. Kochanek., \& Elizabeth, Arias. (2018). Mortality in the United States, Data Brief, No. 335, (January 2020). https://www.cdc.gov/nchs/data/ databriefs/db355-h.pdf

4. Population Reference Bureau, (2020). The US Population Is Growing Older, and the Gender Gap in Life Expectancy Is Narrowing. https://www.prb.org/the-u-s-population-isgrowing-older-and-the-gender-gap-in-life-expectancy-isnarrowing/

5. Jacob, Ausubel. (2020). Pew Research Center, Older People are more likely to live alone in the US than anywhere else in the World. https://www.pewresearch.org/fact-tank/2020/03/10/ older-people-are-more-likely-to-live-alone-in-the-u-s-thanelsewhere- in-the-world/\#: :text=In\%20the\%20U.S.\%2C\%20 $27 \% 25 \% 20$ of, $130 \% 20$ countries $\% 20$ and $\% 20$ territories $\% 20$ studied.

6. Jed, Montayre., Jasmine, Montayre., \& Sandra Thaggard. (2019). The Elder Orphan in Healthcare Settings: An Integrative Review. 12, Population Ageing, 515-532.

7. Maria T. Carney., Janice, Fujiwara., Brian E. Emmert., Tara, A. (2016). Liberman, Barbara Paris, Elder Orphans Hiding in Plain Sight: A Growing Vulnerable Population,"Current Gerontology \& Geriatrics Res. https://doi.org/10.1155/2016/4723250

8. National Academies of Sciences, Engineering, and Medicine, Social Isolation and Loneliness in Older Adults: Opportunities for the Health Care System (2020). https://doi.org/10.17226/25663.

9. CDC, Subjective Cognitive Decline (2018). https://www.cdc. gov/aging/data/subjective-cognitive-decline-brief.html

10. Elena, P., Robert, L Rubinstein., Kenneth, E Covinsky., Jodi, Halpern., \& Julene, K Johnson. (2019). The Precarity of Older Adults Living Alone With Cognitive Impairment, 59, The Gerontologist, 271 - 280. https://doi.org/10.1093/geront/ gnx193

11. Holly, Ramsey-Klawsnik. (2014). The Complexities of Cognitive Capacity. http://www.napsa-now.org/wp- content/ uploads/2015/06/TA-Brief-Mental-Capacity-FINAL.pdf

12. Philip, D. Harvey. (2019). Domains of cognition and their assessment. 21, Dialogues In Clinical Neuroscience, 227 - 237 (2019), DOI:10.31887/DCNS.2019.21.3/PHARVEY

13. Daniel L. Murman. (2015). The Impact of Age on Cognition. 36, Seminars In Hearing, 111-121, DOI:10.1055/s-0035-1555115.

14. National Institute on Aging. (2020). Assessing Cognitive Impairment in Older Patients. https://www.nia.nih.gov/health/ assessing-cognitive-impairment-older-patients 
15. US Preventive Services Task Force. (2020). Screening for Cognitive Impairment in Older Adults: US Preventive Services Task Force Recommendation Statement. JAMA. 323,757-763. DOI:10.1001/jama.2020.0435

16. Kotagal, Vikas., et al. (2015). Factors associated with cognitive evaluations in the United States. Neurology. 84, 64-71 (2015). doi:10.1212/WNL.0000000000001096

17. Sally, McSwiggan., Susanne, Meares., \& Melanie, Porter. (2016). Decision-Making Capacity Evaluation in Adult Guardianship: A Systematic Review. International Psychogeriatrics. 28, 373.

18. Melissa, C. Brouwers., Ivan, D. Florez., Sheila, A. McNair., Emily T. Vella., \& Xioamei, Yao. (2019). Clinical Practice Guidelines: Tools to Support High-Quality Patient Care, Seminars In Nuclear Medicine, 49, 145-152, https://doi. org/10.1053/j.semnuclmed.2018.11.001.

19. Howard, Smith. (2005). A Model For Validating An Expert's Opinion In Medical Negligence Cases, J. Of Legal Med., 26, 207-231. DOI: 10.1080/01947640590949931

20. Allison, Grady. (2005). The Importance of Standard of Care and Documentation. Virtual Mentor. 7,756-758. doi: 10.1001/ virtualmentor.2005.7.11.hlaw1-0511.

21. Freddi, Segal-Gidan. (2013). Cognitive Screening Tools. Clinician Reviews, 23, 12-18.
22. John, E. Morley, et al. (2015). Brain health: the importance of recognizing cognitive impairment: an IAGG consensus conference. J. Of The Am. Med. Directors Assn, 16, 731- 739. DOI:10.1016/j.jamda.2015.06.017

23. Jennifer, Moye., Stacey, Wood., Barry, Edelstein., Jorge, C., Armesto, Emily H. Bower, Julie A. Harrison., \& Erica, Wood. (2007). Clinical Evidence in Guardianship of Older Adults Is Inadequate: Findings From a Tri-State Study, The Gerontologist, 47, 604-612. (October 2007). https://doi. org/10.1093/geront/47.5.604

24. Felicia, Skelton., Mark, E. Kunik., Regev, Tziona., \& Aanand, D. Naik. (2010). Determining if an older adult can make and execute decisions to live safely at home: a capacity assessment and intervention model. Archives of Gerontology \& Geriatrics, 50, 300- 305, (2010). doi:10.1016/j.archger.2009.04.016

25. Kub, J.E., Kulbok, P.A., Miner, S., Merrill, J.A. (2017). Increasing the capacity of public health nursing to strengthen the public health infrastructure and to promote and protect the health of communities and populations. Nurs Outlook. 65(5):661-664. doi:10.1016/j.outlook.2017.08.009

26. Grady, Patricia A. (2011). Advancing the health of our aging population: a lead role for nursing science. Nursing outlook vol. 59,(4): 207-9. doi:10.1016/j.outlook.2011.05.017 